\title{
Über das Verhalten von Potenzreihen mit zwei und drei Veränderlichen an der Konvergenzgrenze.
}

\author{
Von Hermann Holzberger in München.
}

\section{Einleitung.}

Das Verhalten der Potenzreihen einer Variabeln auf dem Rande des Konvergenzkreises ist in den letzten Jahrzehnten eingehend untersucht worden. Im Gegensatz hiezu weif man ïber das Verhalten mehrfacher Reihen auf dem Rande des Konvergenzbereiches noch sehr wenig. Es sind mir hierüber nur einige Arbeiten von B romwich und Hardy bekannt. ${ }^{1}$ ) In der Arbeit "Some extensions...", pag. 164-168, geben die Autoren Erweiterungen des sogenannten Abelschen Grenzwertsatzes auf mehrfache Reihen für einen sehr wichtigen, aber freilich nur speziellen Fall :

1. Konvergiert $\sum_{\mu, v=0}^{\infty} a_{\mu}^{\nu}$ und ist $\left|\sum_{\mu, \nu=0}^{m, n} a_{\mu}^{\nu}\right|<g$ für alle $m$ und $n$ (die Autoren nennen diese Bedingung die der Endliehkeit), so konvergiert $\sum_{\mu, \nu=0}^{\infty} a_{\mu}^{\nu} x^{\mu} y^{v}$ absolut für $|x|<1,|y|<1$ und es ist

$$
\lim _{x, y=1} \sum_{\mu, \nu=0}^{\infty} a_{\mu}^{v} x^{\mu} y^{\nu}=\sum_{\mu, v=0}^{\infty} a_{\mu}^{v}
$$

2. Konvergiert $\sum_{\nu=0}^{\infty} \sum_{\mu=0}^{\infty} a_{\mu}^{v}$ und ist $\left|\sum_{\mu, v=0}^{m, n} a_{\mu}^{v}\right|<g$ für alle $m$ und $n$, so konvergiert $\sum_{\mu, \nu=0}^{\infty} a_{\mu}^{\nu} x^{\mu} y^{\nu}$ absolut für $|x|<1,|y|<1$

1) Hardy, On the convergence of certain multiple series. Proceed. Lond. Math. Soc. (2), 1, 1903/4. - Bromwich and Hardy, Some extensions to multiple. series of Abels theorem on the continuity of power series. Proceed. Lond. Math. Soc. (2), 2, 1904. - Bromwich, Various extensions of Abels lemma. Proceed. Lond. Math. Soc. (2), 6, 1908. 
und es ist

$$
\lim _{y=1} \lim _{x=1} \sum_{\mu, \nu=0}^{\infty} a_{\mu}^{\nu} x^{\mu} y^{\nu}=\sum_{\nu=0}^{\infty} \sum_{\mu=0}^{\infty} a_{\mu}^{\nu} .
$$

Diese beiden Sätze erfahren in der zitierten Abhandlung noch weitere Verallgemeinerung auf $n$-fache Reihen. Bromwich und Hardy machen jedoch dabei immer die beiden Voraussetzungen:

a) Die vorgelegte Reihe erfüllt die Bedingung dèr Endlichkeit. Werte.

b) Die Variabeln durchlaufen beim Grenzübergang nur reelle

Die vorliegende Arbeit beschäftigt sich im ersten Kapitel, $\$ \S 1-3$, damit, unter Verzicht auf die Voraussetzungen a) und $b$ ), die Grenzwertgleichung des Satzes 1 in möglichster Allgemeinheit zu erhalten. Zunächst wird in $\$ 2$ für den Satz 1 unter Beibehaltung der Voraussetzung a) ein neuer Beweis gegeben, der auf der gleichmäßigen Konvergenz der Doppelreihe $\sum_{\mu, v=0}^{\infty} a_{\mu}^{\nu} x^{\mu} y^{\nu}$ in einem gewissen, näher zu bestimmenden Bereiche beruht und uns insoferne ${ }^{1}$ ) einen tieferen Einblick in das Verhalten der Reihe gewährt, als dies bei dem Bromwich-Hardyschen Beweise der Fall ist. Bei dem Grenzübergange sind jedoch die Variabeln in dem Einheitskreise nicht ganz willkürlich, sondern durch die Ungleichungen $\frac{|1-x|}{1-|x|}<g$, $\frac{|1-y|}{1-|y|}<g_{1}$ auf einen bestimmten Bereich beschränkt.

In $\& 3$ wird zum allgemeinen Falle übergegangen, indem man auch die Bedingung a) streicht. Es wird gezeigt, dafo die notwendige und hinreichende Bedingung für die Gleichung $\lim _{x, y=1} \sum_{\mu, \nu=0}^{\infty} a_{\mu}^{\nu} x^{\mu} y^{\nu}=\sum_{\mu, v=0}^{\infty} a_{\mu}^{\nu}$ darin besteht, daß alle Zeilen und Kolonnen der Doppelreihe in der Umgebung des Punktes $x=1$, $y=1$ unter endlicher Grenze bleiben, soferne man schon weib, daß die Reihe $\sum_{\mu, \nu=0}^{\infty} a_{\mu}^{v} x^{\mu} y^{\nu}$ für $|x|<1,|y|<1$ absolut konvergiert. Diese letztere Frage ist aber schon von Herrn $\mathrm{H}$ artog $\mathrm{s}$ beantwortet worden, so daß wir uns nicht mehr mit ihr beschäftigen müssen. Auch für den Fall, daß $\sum_{\mu, v=0}^{\infty} a_{\mu}^{v} x^{\mu} y^{\nu}$ nicht den ganzen Bereich $|x|<1$, $|y|<1$ zum Konvergenzgebiet hat, werden unter Zuhilfenahme Hartogsscher Sätze noch hinreichende Bedingungen für die Grenzwertgleichung gefunden. Endlich wird gezeigt, daß in dem

1) Vgl. Pringsheim, Münch. Ber. 27 (1897), p. 344. 
allgemeinen Falle, in dem eine gewisse Anzahl von Zeilen und Kolonnen in der Umgebung des Punktes $x=1, y=1$ über alle Grenzen wächst, die Reihe $\sum_{\mu, \nu=0}^{\infty} a_{\mu}^{v} x^{\mu} y^{\nu}$ in der Umgebung dieses

Punktes nicht unter endlicher Grenze bleibt, dal sie aber durch Addition eines aus den nicht endlich bleibenden Zeilen und Kolonnen gebildeten Ausdrucks in eine Reihe übergeht, die $\sum_{\mu, \nu=0}^{\infty} \alpha_{\mu}^{\nu}$ zum Grenzwert hat, ein Ergebnis, welches dann als „allgemeiner Abelscher Grenzwertsatz" bezeichnet wird.

Die folgenden Paragraphen (4 und 5) bringen sodann die Erweiterung der Ergebnisse auf den Fall, daß $\sum_{\mu, \nu=0}^{\infty} a_{\mu}^{v}$ nicht konvergiert, wohl aber ein arithmetisehes Mittel besitzt. Bekanntlich hat Höld er zuerst eine Definition des arithmetischen Mittels höherer Ordnung einer unendlichen Reihe gegeben und bewiesen, $\operatorname{da} \beta \lim _{x=1} \sum_{v=0}^{\infty} a_{v} x^{v}=s$, wenn $s$ das arithmetische Mittel der Reihe ist, welchen Satz Bromwich und Hardy anf $n$-fache Reihen übertragen haben. Eine von der Hölderschen abweichende Definition des arithmetischen Mittels höherer Ordnung gab Cesàro; seine Definition, welche bekanntlich von gleicher 'Tragweite wie die Höldersche ist, hat vor dieser den Vorzug, leichter anwendbar zu sein und gestattet eine bedeutende Vereinfachung im Beweise des Grenzwertsatzes. Dies berechtigte uns, von der Hölderschen Definition ganz abzusehen und die Cesàrosche zu Grunde zu legen.

Um aber der vollen Alloemeinheit sicher zu sein, erwies es sich als notwendig, zunächst einen unter dem Namen "Cauchyscher Grenzwertsatz" bekannten Hilfssatz auf Doppelreihen auszudehnen, und dann allgemein nicht nur das arithmetische Mittel erster Ordnung einer Doppelreihe, sondern auch das von beliebiger Ordnung einer genaueren Untersuchung zu unterziehen ( $\$ \$ 4$ und $5,1-2$ ). Es zeigte sich, daß bei einer Reihe, die die Bedingung der Endlichkeit nicht erfüllt, die Definition des arithmetischen Mittels mit einer gewissen Vorsicht zu geschehen hat und daß man eventuell diejenigen Partialsummen $\sum_{\mu, \nu=0}^{m, n} a_{\mu}^{\nu}$, deren Indizes $m, n$ zwei Zablen $M, N$ nicht gleichzeitig übertreffen, weglassen können muß. Ferner wird der Begriff des normalkonvergenten Mittelwertes aufgestellt, $d . \mathbf{h}$. desjenigen Mittelwertes einer Reihe, dessen Näherungswerte alle unter endlicher Grenze bleiben und gezeigt, dab jede Reihe, die einen arithmetischen Mittelwert besitzt, so abgeändert werden kann, daß sie einen normalkonvergentẹn Mittelwert erhält. 
Nunmehr wird für normalkonvergente Mittelwerte der Satz bewiesen:

Besitzt $\sum_{\mu, \nu=0}^{\infty} a_{\mu}^{\nu}$ einen normalkonvergenten Mittelwert $s$, so ist für $|x|<1,|y|<1 \sum_{\mu, \nu=0}^{\infty} a_{\mu}^{v} x^{\mu} y^{\nu}$ absolut konvergent und es gilt (in einem bestimmten Bereiche)

$$
\lim _{x, y=1} \sum_{\mu, \nu=0}^{\infty} a_{\mu}^{v} x^{\mu} y^{v}=s,
$$

von welchem Satz man dann auf Grund der ausgeführten Untersuchungen über arithmetische Mittel von Doppelreihen auf den allgemeinen Fall eines beliebigen, $d$. h. nicht normalkonvergenten Mittelwerts ebenso übergeht wie in $\$ 3$ von der Bedingung a) zum Falle einer beliebigen konvergenten Doppelreihe. Infolgedessen sind alle Ergebnisse von $\$ 3$ auf unser erweitertes Problem naheru wörtlich übertragbar und dieses ebenfalls allgemein gelöst.

In einer Anmerkung werden schließlich noch einige Verallgemeinerungen des Satzes 2 , die leicht zu beweisen sind, erwähll leider war es mir hier nicht möglich, die Grenzen für den Satz 2 durch Angabe notwendiger und hinreichender Bedingungen genau abzustecken.

Das zweite Kapitel bringt nun die Verallgemeinerung der Hauptergebnisse des ersten Kapitels auf dreifache Reihen. Während unter Beibehaltung der Bedingung a) die Verhältnisse sogar für $n$-fache Reihen durchaus ebenso liegen, wie bei Doppelreihen und die einzige Komplikation in der größeren Zahl der Variabeln liegt, treten bei der Untersuchung des allgemeinen Falles schon für $n=3$ Erscheinungen zu Tage, die von den bei Doppelreihen gefundenen durchaus verschieden sind. Es ist nämlich bei dreifachen Reihen durchaus nicht mehr notwendig für den Grenzwertsatz, dafo alle in der dreifachen Reihe enthaltenen zwei- und einfachen Reihen in der Umgebung des Punktes $x_{1}, x_{2}, x_{3}=1$ endlich bleiben; vielmehr kann eine endliche Anzahl zweifacher und sogar eine unendliche Anzahl einfacher Reihen in der Nähe dieses Punktes iiber alle Grenzen wachsen, ohne dafo die Gleichung

$$
\lim _{x_{1}, x_{2}, x_{3}=1} \sum a_{\mu_{1}, \mu_{2}, \mu_{3}} x_{1}^{\mu_{1}} x_{2}^{\mu_{2}} x_{3}^{\mu_{3}}=\sum a_{\mu_{1}, \mu_{2}, \mu_{3}}
$$

beeinträchtigt wird. Doch ist dieses Unendlichwerden der zwei- und einfachen Reihen einer gewissen Beschränkung unterworfen, die mit Hilfe einer Klassifizierung zweifacher Reihen exakt ausgesprochen 
werden kann. Als Resultat ergibt sich, daß die notwendige und hinreichende Bedingung für die Gleichung

$$
\lim _{x_{1}, x_{2}, x_{3}=1} \sum a_{\mu_{1}, \mu_{2}, \mu_{3}} x_{1}^{\mu_{1}} x_{2}^{\mu_{2}} x_{3}^{\mu_{3}}=\sum a_{\mu_{1}, \mu_{2}, \mu_{3}}
$$

darin besteht, daß die dreifache Reihe selbst in der Umgebung des Punktes $x_{1}, x_{2}, x_{3}=1$ unter endlicher Grenze bleibt, ein Resultat, welches ohne weiteres auf den Fall ausgedehnt werden kann, daß die Reihe ein arithmetisches Mittel beliebiger Ordnung besitzt.

Das letzte Kapitel endlich ist der Umkehrung des Abelschen. Grenzwertsatzes gewidmet. Bekanntlich hat Tauber ${ }^{1}$ ) für einfache Reihen den wichtigen Satz bewiesen:

$$
\text { Ist } \lim _{x=1} \sum_{\nu=0}^{\infty} a_{\nu} x^{v}=s \text { und } \lim _{n=\infty} \frac{1 \cdot a_{n}+2 \cdot a_{1}+\cdots(n+1) a_{n}}{n+1}=0,
$$

welche Bedingungen beide zur Konvergenz von $\sum_{\nu=0}^{\infty} a_{\nu}$ notwendig sind; so ist $\sum_{v=0}^{\infty} a_{v}$ konvergent.

Es ist mir gelungen, diesen Satz auf Doppelreihen auszudehnen. Dabei habe ich eine etwas allgemeinere, von Herrn Landa u herrührende Form des Tauberschen Satzes zu Grunde gelegt. $\$ 9$ behandelt den einfachsten Fall, daß $\left(\alpha_{\mu}^{\nu}=\alpha_{\mu}^{v}+i \beta_{\mu}^{\nu}\right.$ gesetzt) alle $\alpha_{\mu}^{\nu}$ als auch alle $\beta_{\mu i}^{\nu}$ unter sich gleiches Zeichen besitzen. Der folgende Paragraph dient der Herleitung notwendiger Konvergenzbedingungen, die der Tauberschen Gleichung $\lim _{n=\infty} \frac{1 . a_{0}+2 \cdot a_{1}+\cdots(n+1) a_{n}}{n+1}=0$ entsprechen. Im letzten Paragraph endlich wird die Übertragung des Tauber-Landauschen Satzes zuerst unter einer der Bedingung a) sehr nahe verwandten Voraussetzung ausgeführt und dann mit Hilfe der Ergebnisse von $\$ \S 3$ und. 9 auf beliebige Doppelreihen ausgedehnt.

1) Monatsh. für Math. n. Physik VIIL, 1897, p. 273. - Pringsheim, Miinch. Ber. 30,1900 , p. 51. 